\title{
Effects of the LHPP gene polymorphism on the functional and structural changes of gray matter in major depressive disorder
}

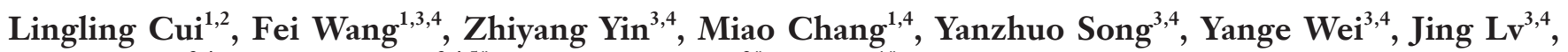 \\ Yifan Zhang ${ }^{3,4}$, Yanqing Tang ${ }^{3,4,5 \#}$, Xiaohong Gong ${ }^{2 \#}, \mathrm{Ke} \mathrm{Xu}^{1 \#}$ \\ ${ }^{1}$ Department of Radiology, The First Affiliated Hospital of China Medical University, Shenyang 110001, China; ${ }^{2}$ State Key Laboratory of Genetic \\ Engineering and MOE Key Laboratory of Contemporary Anthropology, School of Life Sciences, Fudan University, Shanghai 200433, China; \\ ${ }^{3}$ Department of Psychiatry, ${ }^{4}$ Brain Function Research Section, ${ }^{5}$ Department of Geriatrics, The First Affiliated Hospital of China Medical University, \\ Shenyang 110001, China
}

\#These authors contributed equally to this work.

Correspondence to: Ke Xu, PhD. Department of Radiology, The First Affiliated Hospital of China Medical University, 155 Nanjing North Street, Heping District, Shenyang 110001, China. Email: kxu@cmu.edu.cn; Xiaohong Gong, PhD. State Key Laboratory of Genetic Engineering and MOE key Laboratory of Contemporary Anthropology, School of Life Sciences, Fudan University, Shanghai 200433, China. Email: gongxh@fudan.edu.cn; Yanqing Tang. Department of Geriatrics and Psychiatry, The First Affiliated Hospital, China Medical University, 155 Nanjing North Street, Heping District, Shenyang 110001, China. Email: yanqingtang@163.com.

Background: A single-nucleotide polymorphism (SNP) of the LHPP gene (rs35936514) has been reported to be associated with major depressive disorder (MDD) in genome-wide association studies. However, the systems-level neural effects of rs35936514 that mediate the association are unknown. We hypothesized that variations in rs35936514 would be associated with structural and functional changes in gray matter (GM) at rest in MDD patients.

Methods: A total of 50 MDD patients and 113 healthy controls (HCs) were studied. Functional connectivity (FC) was analyzed by defining the bilateral hippocampus as the seed region. Voxel-based morphometry (VBM) was performed to assess the patterns of GM volume. The subjects were further divided into two groups: a CC homozygous group (CC; $24 \mathrm{MDD}$ and $56 \mathrm{HC}$ ) and a risk T-allele carrier group (CT/ TT genotypes; $26 \mathrm{MDD}$ and $57 \mathrm{HC}$ ). A $2 \times 2$ analysis of variance (ANOVA: diagnosis $\times$ genotype) was used to determine the interaction effects and main effect $(\mathrm{P}<0.05)$.

Results: Significant diagnosis $\times$ genotype interaction effects on brain morphology and FC were noted. Compared to other subgroups, the MDD patients with the $\mathrm{T}$ allele showed an increased hippocampal FC in the bilateral calcarine cortex and cuneus and a decreased hippocampal FC in the right dorsolateral prefrontal cortex (DLPFC), bilateral anterior cingulate cortex (ACC), and medial prefrontal cortex (MPFC), in addition to reduced GM volume in the right DLPFC, bilateral temporal cortex, and posterior cingulate cortex (PCC). Conclusions: LHPP gene polymorphisms may affect functional and structural changes in the GM at rest and may play an important role in the pathophysiological mechanisms of MDD.

Keywords: Major depressive disorder (MDD); functional connectivity (FC); voxel-based morphometry (VBM); LHPP; genetic polymorphism

Submitted Jun 29, 2019. Accepted for publication Nov 25, 2019.

doi: $10.21037 /$ qims.2019.12.01

View this article at: http://dx.doi.org/10.21037/qims.2019.12.01 


\section{Introduction}

Major depressive disorder (MDD) is characterized by high rates of recurrence, disability, and suicide $(1,2)$, and the diagnostic criteria rely only on clinical symptomatology, without the clinical use of biological markers. The genetic characteristics of depression are complex, and these genes cannot be used to diagnose the disease or to explain the clinical symptoms directly. However, imaging genetics has provided new evidence in the study of mental diseases $(3,4)$.

At present, imaging genetics has verified that risk genes affect the normal structure and function of the neural system in the brain (3,5-9). The rs35936514 singlenucleotide polymorphism (SNP) of the LHPP gene has been reported to be significantly associated with MDD at the genome-wide level (10). LHPP encodes an enzyme known as phospholysine phosphohistidine inorganic pyrophosphate phosphatase (LHPP), which shows high levels of expression in the brain $(11,12)$. This region of the genome has been implicated in the etiology of MDD using a combination of linkage and association analysis (12). However, relatively little is known about the mechanism of LHPP involvement in depression.

In previous studies, we have reported differences in brain activation between the variant carriers in the classic emotion-related brain regions, in the primary cortex, and in other brain regions of MDD patients (13) that cause pathological and physiological changes associated with MDD. Previous studies have shown that the expression level of LHPP is related to thyroid function (14), and thyroid diseases have also been widely indicated to be related to depression (15). LHPP is more prominent in hyperfunctional states of the thyroid, such as those occurring in Graves' disease and autonomously functioning thyroid nodule (AFTN), than in other states. Since these cell types have specific functions, it seems likely that LHPP may be more related to thyrocyte-specific thyroid hormone (TH) synthesis than to the various functions of endothelial cells, fibroblasts, and adipocytes (14).

Studies have shown that any thyroid dysfunction will seriously affect the central nervous system and cause changes in brain morphology and function, resulting in a variety of neurological abnormalities (16-23). TH is critical for the overall architecture of the human brain during early development and for normal brain function throughout life $(24,25)$. Severe restrictions of TH undermine these developmental processes in the brain and lead to various neurological dysfunctions (16-19), with the hippocampus being particularly sensitive to $\mathrm{TH}$ restriction (26), as it exhibits morphological plasticity throughout adult life $(27,28)$. Therefore, we speculated that the polymorphism of the LHPP gene may be related to the structure and function of the hippocampal-cortical neural system in MDD patients and may participate in the pathophysiological mechanisms that drive MDD symptoms.

Functional connectivity (FC) analysis investigates the functional integration of brain regions, including not only the abnormal activation of brain regions but also the abnormal functional integration between brain regions that occur in depression. Resting-state FC (rsFC) can explore the connection between these regional functional networks (29) more comprehensively and specifically reveal the pathophysiological changes of mental diseases $(30,31)$. Studies have reported abnormal FC within the nervous system in MDD patients $(32,33)$ and have shown that changes in the hippocampus are related to the pathophysiological mechanisms of MDD (34-38); therefore, we aimed to study whether a specific LHPP gene polymorphism was associated with hippocampal-cortical neural circuitry. Voxel-based morphometry (VBM) (39) is a technique based on $3 \mathrm{D}$ T1-weighted images and is used to compare brain volume among groups. VBM does not require any a priori assumptions or images of the whole brain. Over the past decade, this technique has drawn increasing attention and has been used to investigate reduced gray matter (GM) volume in $\operatorname{MDD}(39,40)$.

VBM and FC analyses have revealed several GM structural and functional abnormalities related to cognitive impairment in MDD patients. The effects of the neural system and their association with LHPP variation in the context of MDD are not yet understood. Exploration of the related physiological pathways is of great significance for the diagnosis and treatment of depression. Therefore, the purpose of this study was to further investigate the relationship between allelic variation at rs35936514 and both the structural and functional changes of the hippocampal-cortical circuitry in MDD patients. We hypothesized that the polymorphism rs35936514 might increase the risk of MDD by driving structural and functional alterations.

\section{Methods}

\section{Participants}

A total of 163 Chinese subjects were studied. Fifty MDD 
patients (age: mean \pm SD $27.66 \pm 12.17$ years, $70 \%$ female) were recruited from the outpatients at the Department of Psychiatry at the First Hospital of China Medical University and the Mental Health Center of Shenyang. The diagnosis of MDD was confirmed by 3 trained psychiatrists using the Structured Clinical Interview for DSM-IV disorders (SCID) (41). Scores on the 17-item Hamilton Depression Rating Scale (HAMD) were obtained from all participants, except for one patient who did not complete these evaluations (42). A total of 113 age- and sex-matched healthy controls (HCs) (age: mean \pm SD $33.37 \pm 14.08$ years, $58 \%$ female) were recruited from the community. No personal history of a DSM-IV Axis I Disorder (41) was found in the healthy individuals or in their first-degree family members, as assessed by the Family History Screen for Epidemiologic Studies (43). HCs had no history of psychiatric or neurological illness, psychiatric treatment, drug or alcohol abuse, or head trauma with a loss of consciousness $\geq 5 \mathrm{~min}$, and they had no contraindications to magnetic resonance imaging (MRI) examination. Subjects had not used substances in the week prior to scanning, and the urine toxicology screens on the day of scanning were negative. All of the participants provided written informed consent after receiving a complete description of the study. This study was approved by the Medical Research Ethics Committee of China Medical University.

\section{Genotyping}

Ten-milliliter blood samples were collected from all of the participants from the neuroimaging study for DNA extraction. The rs35936514 polymorphism was genotyped using Sanger sequencing methods. The forward primer of rs35936514 was 5'-TTC AGG CCC CTA AAC TTG TG-3', and the reverse primer was 5'-CCA GCA ATT CCA TGT GTG AG-3'. We divided the subjects into two groups according to the rs35936514 genotype: the homozygous group for the $\mathrm{C}$ allele (CC genotype, $\mathrm{n}=80$, mean age $=33.16 \pm 14.08$ years, $61.3 \%$ female $)$ and the T-allele carrier group (CT/TT genotypes, $n=83$, mean age $=30.14 \pm 12.93$ years, $61.4 \%$ female) .

\section{MRI scan acquisition}

All of the participants were scanned using a GE Signa HDx 3.0T MRI scanner at the First Hospital of China Medical University in Shenyang, China. Functional images were obtained using an echo-planar imaging (EPI) sequence
$\left(\mathrm{TR}=2,000 \mathrm{~ms}, \mathrm{TE}=30 \mathrm{~ms}\right.$, matrix $=64 \times 64$, flip angle $=90^{\circ}$, field of view $(F O V)=240 \times 240 \mathrm{~mm}$, slice thickness $=3 \mathrm{~mm}$, 35 slices without gap). Finally, three-dimensional T1weighted structural images were obtained using a fast spoiled gradient-echo (SPGR) sequence ( $\mathrm{TR}=7.1 \mathrm{~ms}$, $\mathrm{TE}=3.2 \mathrm{~ms}$, matrix $=240 \times 240, \mathrm{FOV}=240 \times 240 \mathrm{~mm}$, slice thickness $=1.0 \mathrm{~mm}, 176$ slices without gap). The subjects were asked to keep their eyes closed but not to fall asleep or to think systematically throughout the scan.

\section{Structural data preprocessing}

Structural brain images were processed using the VBM8 toolbox (http://dbm.neuro.uni-jena.de/vbm8/), which was incorporated into the statistical parametric mapping (SPM) software (SPM8; The Wellcome Department of Cognitive Neurology). First, all of the subjects' anterior commissures (ACs) were coregistered to the central point of space. Then, the segmentation function was performed to divide the regions into the cerebrospinal fluid (CSF), white matter (WM), and GM partitions using the "New Segment" tool implemented in SPM8 (44). During spatial normalization, intersubject registration was achieved using respective registration based on a group assignment. A modulation step was used to ensure that the overall amount of tissue in a class was unaltered. The segmented images were performed to match the corresponding Montreal Neurological Institute (MNI) templates (http://www.mni.mcgill.ca/). Finally, all images were smoothed using an isotropic Gaussian kernel with an 8 -mm full width at half-maximum (FWHM). These segmented, normalized, modulated, and spatially smoothed GM images were then used for subsequent VBM statistical analysis.

\section{Rs-fMRI data preprocessing}

Brain functional dates were preprocessed using Data Processing \& Analysis of Brain Imaging (DPABI) (http:// rfmri.org/DPABI) (45) running on MATLAB R2010b (MathWorks Inc., Natick, MA, USA). Resting-state fMRI data preprocessing included discarding the first 10 volumes of each time series, slice timing, head motion correction, and normalization to the MNI template (resampling voxel size $=3 \mathrm{~mm} \times 3 \mathrm{~mm} \times 3 \mathrm{~mm}$ ), followed by the spatial smoothing $(\mathrm{FWHM}=6 \mathrm{~mm})$. Participants with more than $2.5^{\circ}$ of head rotation or with head motion with $>2.5 \mathrm{~mm}$ of maximum displacement in any dimension were excluded. Preprocessing by REST consisted of the filtration of the 
Table 1 Demographics and clinical data of participants

\begin{tabular}{|c|c|c|c|c|c|c|}
\hline Variable & \multicolumn{2}{|c|}{$\operatorname{MDD}(n=50)$} & \multicolumn{2}{|c|}{$\mathrm{HC}(n=113)$} & Value & $\mathrm{P}$ \\
\hline Gender (male/female) & $7 / 17$ & $8 / 18$ & $24 / 32$ & $24 / 33$ & $\chi^{2}=3.52$ & 0.32 \\
\hline Age (years), mean $\pm S D$ & $29.91 \pm 12.97$ & $25.59 \pm 11.43$ & $34.55 \pm 14.55$ & $32.22 \pm 13.62$ & $F=2.39$ & 0.07 \\
\hline Education (years), mean \pm SD & $11.27 \pm 2.41$ & $11.19 \pm 2.89$ & $13.45 \pm 4.09$ & $12.43 \pm 4.45$ & $F=2.60$ & 0.052 \\
\hline HAMD score, mean \pm SD & $20.74 \pm 7.73$ & $21.81 \pm 6.95$ & $1.19 \pm 2.19$ & $1.17 \pm 2.24$ & $F=195.06$ & 0.000 \\
\hline
\end{tabular}

There was no significant effect of diagnosis, genotype, or interaction between diagnosis and genotype for age, sex, or education. Twosample $t$-tests showed no difference in the duration of illness between the MDD subgroups. The genotype had no significant effect on the HAMD score. HAMD scores differed significantly between groups. MDD, major depressive disorder; HC, healthy controls; SD, standard deviation; HAMD, Hamilton Depression Rating Scale.

time series for each voxel (bandpass filtering, 0.01-0.08 Hz) to reduce the effects of high-frequency physiological noise and of low-frequency drifts. A linear regression of the head motion parameters, WM signal, CSF signal, and the global mean signal was performed to remove the effects of the nuisance covariates.

\section{Definition of regions of interest (ROIs)}

The bilateral hippocampus seed ROI was selected according to the definition of the automated anatomical labeling (AAL) template (46) contained in DPABI (resampling voxel size $=3 \mathrm{~mm} \times 3 \mathrm{~mm} \times 3 \mathrm{~mm}$ ). For each participant, a reference time series was extracted by averaging the fMRI time series of all of the voxels within the ROIs. Correlations were computed between the seed ROI and the rest of the brain in a voxel-wise manner using DPABI. The correlation coefficients were transformed to z-values using the Fisher r-to-z transformation.

\section{Statistical analysis}

Statistical Package for the Social Sciences (SPSS) version 20.0 (SPSS Inc., Chicago, IL, USA) software was used to perform statistical analysis of the demographic data. Between-group age, education, and HAMD scores were compared with two-way analysis of variance (ANOVA) with the diagnostic group (HC or MDD) and genotype group (CC or CT/TT) used as the between-subject factors, and pairwise comparison was performed with the Bonferroni method. Between-group sex differences were analyzed using chi-squared tests. A two-sample $t$-test was used to compare the duration of illness across genotypes within the MDD group.

A voxel-wise ANOVA $(2 \times 2$ ANOVA: diagnosis $\times$ genotypes) was used to determine the effects of the diagnosis and genotype on the GM volumes (GMV) and FC strength, and the age and sex were considered to be covariates. The post hoc Bonferroni test was used to examine the details of the main effects and interactions, which were performed with the general linear model (GLM) in SPM8.

\section{Results}

\section{Demographic and genetic data}

There was no significant effect of diagnosis, genotype, or interaction between diagnosis and genotype for age, sex, or education. Two-sample $t$-tests showed no difference in the duration of illness between the two genotype subgroups in MDD. Genotype had no significant effect on HAMD. The groups differed significantly in the HAMD scores, with significantly higher HAMD scores in the MDD group than in the HC group (Table 1).

There was no significant difference in genotype frequency or allele frequency between the MDD group and the HC group (Table 2). Genotype frequencies were consistent with the Hardy-Weinberg equilibrium expectation (MDD: $\chi^{2}=1.957, \mathrm{P}=0.161 ; \mathrm{HC}: \chi^{2}=1.693$, $\mathrm{P}=0.193)$.

\section{VBM analysis}

The VBM analysis revealed a significant diagnosis $x$ genotype interaction in the right dorsolateral prefrontal 
Table 2 Genotype and allele frequency distribution in the MDD and $\mathrm{HC}$ groups

\begin{tabular}{llcccccc}
\hline \multirow{2}{*}{ Group } & \multirow{2}{*}{ Number } & \multicolumn{3}{c}{ Genotype } & & \multicolumn{3}{c}{ Allele } \\
\cline { 3 - 5 } \cline { 6 - 7 } & & CC & CT & TT & & C & T \\
\hline MDD & 50 & 24 & 18 & 8 & & 66 & 34 \\
HC & 113 & 56 & 51 & 6 & & 163 & 63 \\
$\chi^{2}$ & & & 5.312 & & & 1.244 \\
$P$ & & & 0.07 & & & 0.163 \\
\hline
\end{tabular}

There was no significant difference in genotype frequency or allele frequency between the MDD group and the HC group. MDD, major depressive disorder; $\mathrm{HC}$, healthy controls. cortex (DLPFC), bilateral temporal cortex and posterior cingulate cortex (PCC) with a Gaussian random field (GRF) correction for multiple comparisons (voxel level $\mathrm{P}<0.01$ and cluster level $\mathrm{P}<0.05$ ) (Figure 1, Table 3). A post hoc $t$-test revealed that the MDD patients with a $T$ allele showed reduced GM volume among these regions.

Although we focused on the significant interaction effect between the diagnosis and genotype, there were also significant main effects of diagnosis and genotype (GRF correction at voxel level $\mathrm{P}<0.01$ and at cluster level $\mathrm{P}<0.05$ ). The detailed results are provided in Supplementary results, and in Figure $S 1 A, B$, and Table 3.

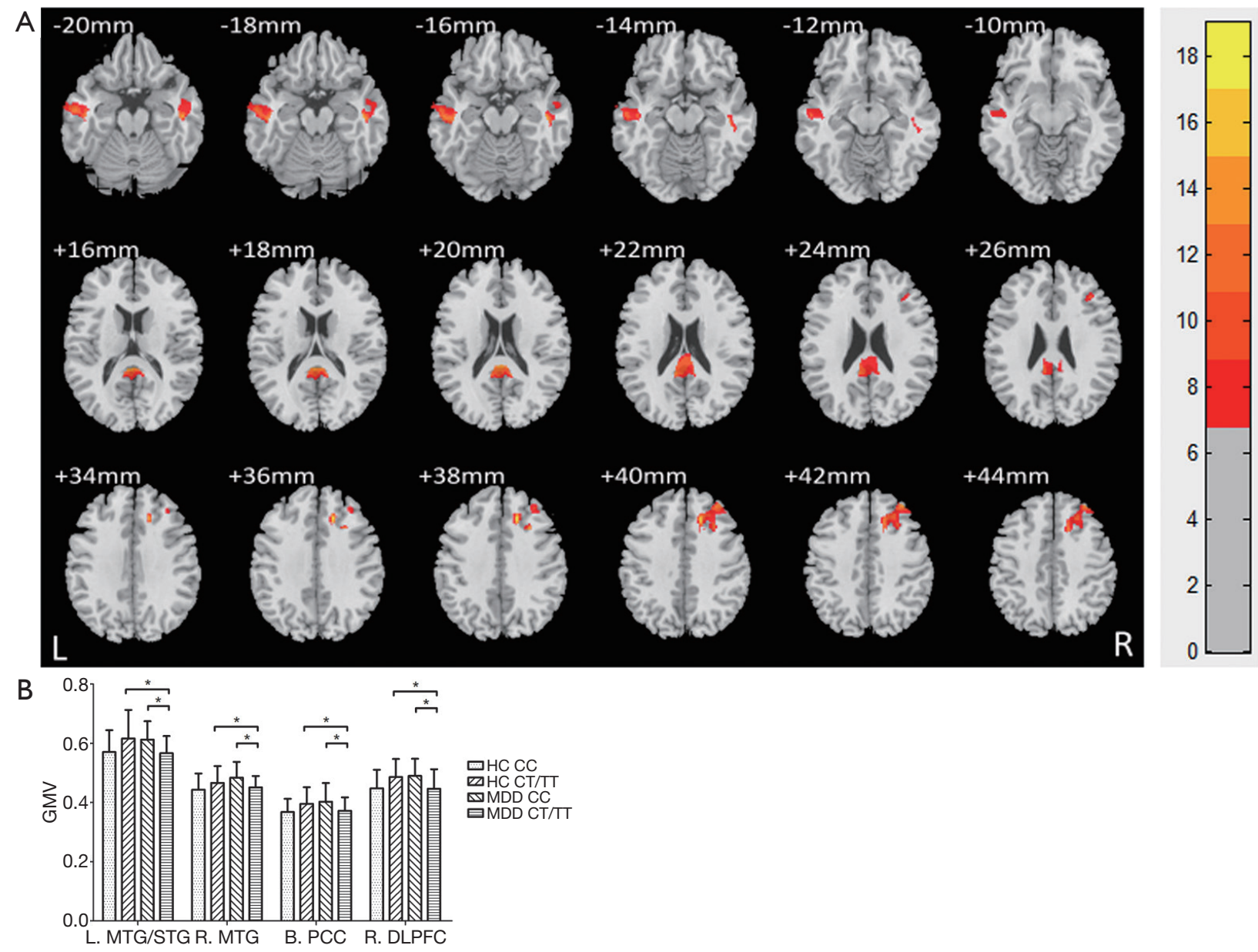

Figure 1 Brain regions with significant diagnosis $\times$ genotype interaction on GMV. (A) Clusters of significance for the interaction effect in the right DLPFC, bilateral temporal cortex, and PCC with a GRF correction for multiple comparisons (voxel level $\mathrm{P}<0.01$ and cluster level $\mathrm{P}<0.05$ ). The color bar represents the range of $\mathrm{F}$ values; (B) an interaction graph showing that the MDD patients with the $\mathrm{T}$ allele showed reduced GM volume among these regions. *, $\mathrm{P}<0.05$. GM, gray matter; GMV, gray matter volume; DLPFC, dorsolateral prefrontal cortex; PCC, posterior cingulate cortex; GRF, Gaussian random field; MDD, major depressive disorder. 
Table 3 Clusters exhibiting the influence of groups and genotypes on GM volume

\begin{tabular}{|c|c|c|c|c|c|c|}
\hline Brain area & $\mathrm{BA}$ & Cluster size & \multicolumn{3}{|c|}{ Peak MNI coordinates } & Peak F value \\
\hline \multicolumn{7}{|l|}{ The main effect of diagnostic groups } \\
\hline Right insula/right STG & $13 / 40 / 41$ & 503 & 51 & -30 & 16.5 & 11.45 \\
\hline Right MTG/occipital cortex & 19 & 635 & 43.5 & -78 & 18 & 13.23 \\
\hline Right STG/temporal pole & $38 / 21$ & 337 & 57 & 10.5 & -13.5 & 12.39 \\
\hline $\begin{array}{l}\text { Right parahippocampal gyrus/hippocampus/ } \\
\text { amygdala/fusiform }\end{array}$ & & 789 & 27 & -9 & -22.5 & 12.30 \\
\hline Right OFC & $47 / 11$ & 539 & 18 & 12 & -22.5 & 12.68 \\
\hline Right MTG & 21 & 446 & 46.5 & -19.5 & -16.5 & 14.52 \\
\hline Bilateral PCC & 23 & 841 & 0 & -39 & 16.5 & 14.41 \\
\hline Right DLPFC & $8 / 9$ & 933 & 16.5 & 28.5 & 37.5 & 18.05 \\
\hline
\end{tabular}

These findings correspond to a corrected $\mathrm{P}<0.05$ by GRF correction. GM, gray matter; BA, Brodmann's area; STG, superior temporal gyrus; MTG, middle temporal gyrus. The cluster size is in $\mathrm{mm}^{3}$.

\section{RsFC analysis}

We found a significant diagnosis $\times$ genotype interaction of the bilateral hippocampus-rsFC in the bilateral calcarine cortex, cuneus, right DLPFC, bilateral anterior cingulate cortex (ACC), and medial prefrontal cortex (MPFC) with a GRF correction for multiple comparisons (voxel level $\mathrm{P}<0.01$ and cluster level $\mathrm{P}<0.05$ ) (Figure 2, Table 4). MDD patients with the $\mathrm{T}$ allele showed increased hippocampalFC in the bilateral calcarine cortex and cuneus and decreased hippocampal-FC in the right DLPFC, bilateral ACC, and MPFC. We did not find any significant main effect of diagnosis or genotype on the rsFC of the bilateral hippocampus (GRF correction at voxel level $\mathrm{P}<0.01$ and at cluster level $\mathrm{P}<0.05)$.

\section{Discussion}

Variation in LHPP rs35936514 is associated with structural and functional changes in the GM within the hippocampalcortical neural system and is implicated in MDD, suggesting that the effects of LHPP variation may contribute to the disorder of the neural system. In a previous study, we demonstrated for the first time that LHPP variation might affect regional brain activity in MDD patients (13). To our knowledge, this is the first study that combined rsFC and VBM to investigate the relationship of LHPP genetic variation with the hippocampal-cortical neural system.

A linkage and sequencing analysis on families with a strong history of MDD identified disease-segregating SNPs in LHPP that interacted with the HTR1A-1019G allele (12). LHPP was then evaluated in a neuroimaging paradigm, and this analysis provided support for the biological function of the risk variant (47). Molecular data can provide a basis for developing important preventive treatments, especially for high-risk categories such as people with substance abuse disorders. Indeed, the LHPP protein product should be a promising target to develop drug therapies, since its enzymatic function could be modulated by inhibitors or stimulators.

Convergent evidence from MRI studies supports the involvement of structural and functional abnormalities in the corticolimbic neural system, including the hippocampus, amygdala, DLPFC, MPFC, ventral prefrontal cortex (VPFC), and cingulate cortex in MDD patients (48-60). Recent research that used structural or functional neuroimaging approaches suggested that the 


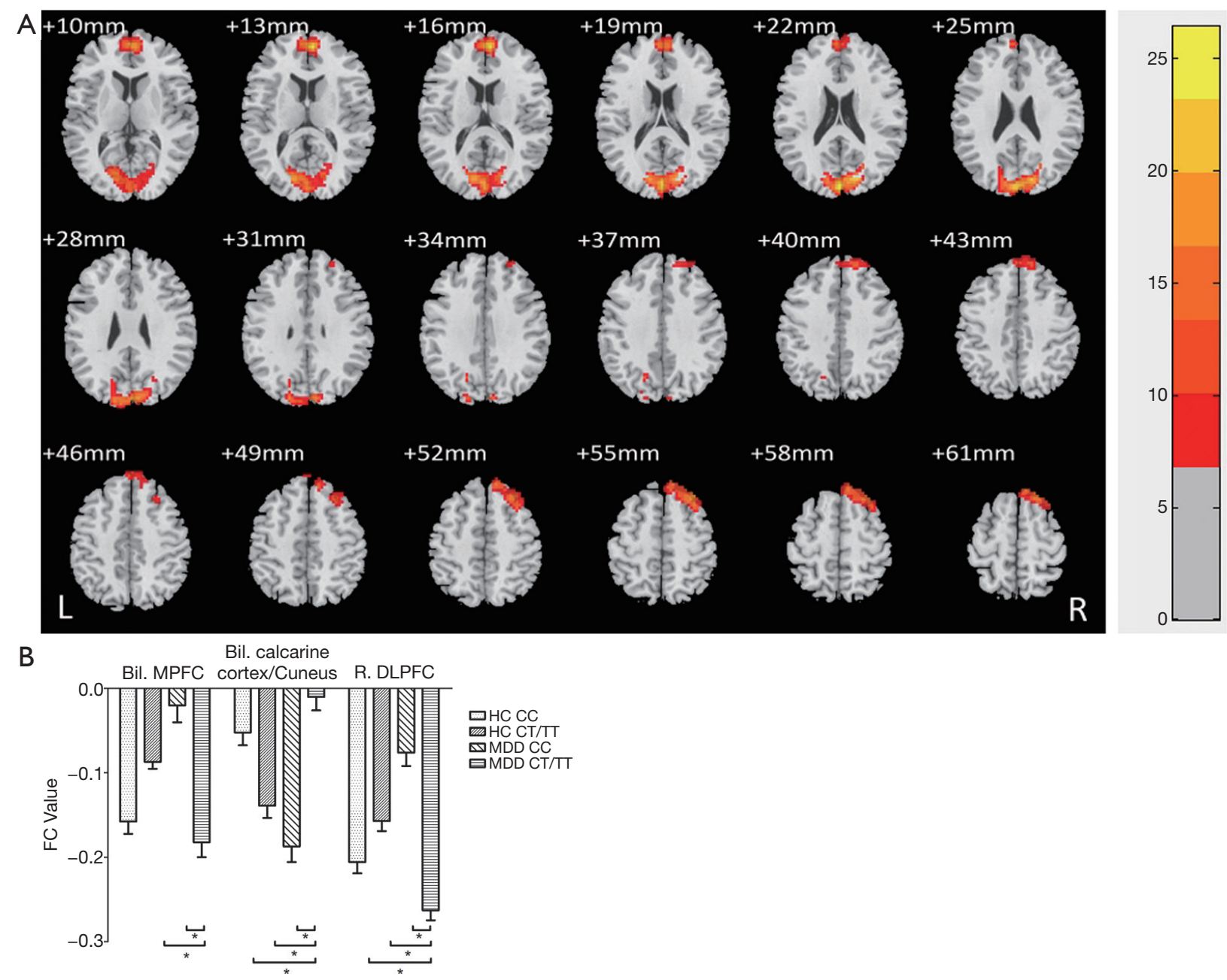

Figure 2 Brain regions with significant diagnosis $\times$ genotype interaction on FC. (A) The FC analysis revealed a significant diagnosis $\times$ genotype interaction of the bilateral hippocampus-rsFC in the bilateral calcarine cortex, cuneus, right DLPFC, bilateral ACC, and MPFC with a GRF correction for multiple comparisons (voxel level $\mathrm{P}<0.01$ and cluster level $\mathrm{P}<0.05$ ); (B) an interaction graph showing that $\mathrm{MDD}$ patients with the $\mathrm{T}$ allele showed an increased hippocampal-FC in the bilateral calcarine cortex and cuneus and a decreased hippocampalFC in the right DLPFC, bilateral ACC, and MPFC. The color bar represents the range of $\mathrm{F}$ values. * $\mathrm{P}<0.05$. FC, functional connectivity; DLPFC, dorsolateral prefrontal cortex; ACC, anterior cingulate cortex; MPFC, medial prefrontal cortex; GRF, Gaussian random field; MDD, major depressive disorder.

Table 4 Clusters exhibiting the group $\times$ genotype interaction effect on $\mathrm{FC}$ values

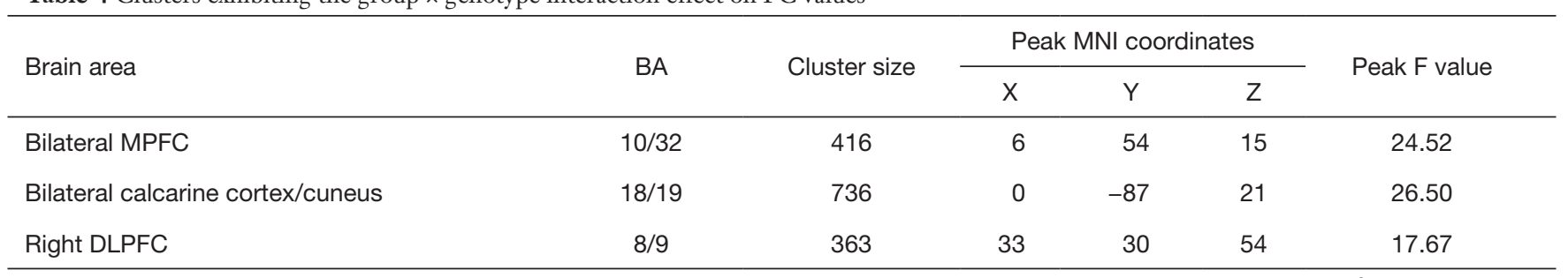

These findings correspond to a corrected $\mathrm{P}<0.05$ by GRF correction. BA, Brodmann's area. The cluster size is in $\mathrm{mm}^{3}$. FC, functional connectivity; MPFC, medial prefrontal cortex; DLPFC, dorsolateral prefrontal cortex. 
dysregulation of both structural and functional changes in the corticolimbic neural system occurs in MDD (61).

In the current study, the major finding was the presence of hippocampal-FC alteration and reduced GM volume in the prefrontal cortex (PFC) of MDD patients with the $\mathrm{T}$ allele. The PFC has an extensive neural junction with other brain regions, such as the other cortical regions, the subcortex, and the brain stem (62). The PFC has advanced executive functions that ensure the overall coordination of the central nervous system (63). The DLPFC is one of the key brain regions that is frequently involved in the pathophysiological mechanisms of MDD; it is involved in emotional regulation, emotional cognition, and other functions (64). The DLPFC is involved in decisionmaking and the cognitive processing of emotions in depression (65). Changes to the cortical-limbic system FC that involve the classical effective brain regions can provide a theoretical basis for the involvement of the LHPP gene polymorphism in emotional processing and in the pathogenesis of MDD.

We also found functional or structural changes in the GM of the MPFC, temporal cortex, and PCC within T-allele carriers in the MDD group. All the brain regions listed above belong to the default mode network (DMN), which suggests that the default mode network is related to the LHPP gene polymorphism and is involved in emotion regulation. When the DMN (66) is without a task, it is in a normal conscious resting state; the brain maintains emotional and cognitive interactions, remains alert to the monitoring of the inside and the outside environment, and shows the highly reactive state of the brain regions. The DMN is the most popular brain resting-state network target; areas including the MPFC, temporal cortex, PCC, precuneus and posterior parietal, and the $\mathrm{DMN}$ are believed to be involved in higher cognitive function (67). The PCC is the core area of structure and function in the DMN and plays an important role in episodic memory. Reduced GM volume in the PCC may affect episodic memory function. The MPFC can receive sensory information from the body and external environment and has close nerve connections with structures such as the hypothalamus, amygdala and ventral striatum. In conclusion, abnormalities in these regions may be involved in emotional and cognitive dysfunction in MDD patients.

Brain regions such as the cuneus and calcarine cortex are involved in the most basic functions, in higher-order visual processing, and in the generation of visual images. In this study, it was found that a change in the hippocampal-visual cortex functional connection was related to polymorphism of the LHPP gene, indicating that the LHPP genotype was important for the functioning of the visual cortex. Recent studies have found that if the maternal TH level is insufficient or too high during the development of the fetus, it will cause synaptic instability of the axon loop in the thalamus cortex, thus affecting the early development of the fetus' visual cortex; this finding provides further evidence for the importance of LHPP in the visual cortex (68). Therefore, more studies are needed to elucidate the effects of the LHPP rs35936514 polymorphism on the neural system in MDD patients and to prove the genetic susceptibility of these neural systems further. Previous reports have shown that there is a correlation between hippocampal-visual cortex abnormality and affective abnormality, revealing that MDD patients show changes in hippocampal-visual cortex pathways $(32,69)$.

Although we focused on the significant interaction effect between diagnosis and genotype, the VBM analysis also revealed significant main effects of diagnosis and genotype. It is possible that variation in disease and genotype may separately contribute to the GM abnormalities in different brain regions in individuals. Both diagnosis and genotype have exhibited effects on brain structure.

Some limitations need to be considered. First, because multiple genes or SNPs are involved in the pathological mechanisms of MDD, the interactions of these genes with the LHPP genotype may also lead to changes in the neural system of MDD patients (70); this needs to be proven through genetic marker imaging in future studies. Many factors may affect the neural system of MDD patients, and it is difficult to explain when and how these interactions occur (71-73). In addition, our study was a cross-sectional study, and a longitudinal study was not performed. Longitudinal studies can provide a deeper understanding of the neural structure, behavior, and clinical manifestations involved in the entire pathogenesis of the disease. Longitudinal studies were not included in this study, which may also limit the interpretation of its results.

\section{Conclusions}

This study suggests that the LHPP rs35936514 polymorphism may play a role in the pathophysiology of neural system abnormalities in patients with MDD. We concluded that the functional and structural changes in the GM that were associated with cognitive impairment in MDD patients were likely to be affected by the LHPP gene polymorphism. 


\section{Acknowledgments}

Funding: This study was supported by the National Key Projects (2016YFC0906400 to X Gong), the National Natural Science Foundation of China Funding support: the National Natural Science Foundation of China (81571311 to Y Tang, 81571331 to F Wang), the National Science Fund for Distinguished Young Scholars (81725005 to F Wang), the National Key Research and Development Program (2016YFC1306900 to Y Tang, 2016YFC0904300 to F Wang), the National High Tech Development Plan (863) (2015AA020513 to F Wang), the Liaoning Science and Technology Project (2015225018 to Y Tang), and the Liaoning Education Foundation (Pandeng Scholar to F Wang).

\section{Footnote}

Conflicts of Interest: The authors have no conflicts of interest to declare.

Ethical Statement: The study protocol was approved by the Institutional Review Board (IRB) of its university and by the local ethics committee.

\section{References}

1. Gotlib IH, Joormann J. Cognition and depression: current status and future directions. Annu Rev Clin Psychol 2010;6:285-312.

2. Bode K, Vogel R, Walker J, Kroger C. Health care costs of borderline personality disorder and matched controls with major depressive disorder: a comparative study based on anonymized claims data. Eur J Health Econ 2017;18:1125-35.

3. Meyer-Lindenberg A, Weinberger DR. Intermediate phenotypes and genetic mechanisms of psychiatric disorders. Nat Rev Neurosci 2006;7:818-27.

4. Scharinger C, Rabl U, Sitte HH, Pezawas L. Imaging genetics of mood disorders. Neuroimage 2010;53:810-21.

5. Canli T, Ferri J, Duman EA. Genetics of emotion regulation. Neuroscience 2009;164:43-54.

6. Phillips ML, Drevets WC, Rauch SL, Lane R. Neurobiology of emotion perception I: The neural basis of normal emotion perception. Biol Psychiatry 2003;54:504-14.

7. Phillips ML, Drevets WC, Rauch SL, Lane R. Neurobiology of emotion perception II: Implications for major psychiatric disorders. Biol Psychiatry 2003;54:515-28.

8. Arslan A. Imaging genetics of schizophrenia in the postGWAS era. Prog Neuropsychopharmacol Biol Psychiatry 2018;80:155-65.

9. Bang M, Kang JI, Kim SJ, Park JY, Kim KR, Lee SY, Park K, Lee E, Lee SK, An SK. Reduced DNA Methylation of the Oxytocin Receptor Gene Is Associated With Anhedonia-Asociality in Women With RecentOnset Schizophrenia and Ultra-high Risk for Psychosis. Schizophr Bull 2019;45:1279-90.

10. CONVERGE consortium. Sparse whole-genome sequencing identifies two loci for major depressive disorder. Nature 2015;523:588-91.

11. Yokoi F, Hiraishi H, Izuhara K. Molecular cloning of a cDNA for the human phospholysine phosphohistidine inorganic pyrophosphate phosphatase. J Biochem 2003;133:607-14.

12. Neff CD, Abkevich V, Packer JC, Chen Y, Potter J, Riley R, Davenport C, DeGrado Warren J, Jammulapati S, Bhathena A, Choi WS, Kroeger PE, Metzger RE, Gutin A, Skolnick MH, Shattuck D, Katz DA. Evidence for HTR1A and LHPP as interacting genetic risk factors in major depression. Mol Psychiatry 2009;14:621-30.

13. Cui L, Gong X, Tang Y, Kong L, Chang M, Geng H, $\mathrm{Xu}$ K, Wang F. Relationship between the LHPP Gene Polymorphism and Resting-State Brain Activity in Major Depressive Disorder. Neural Plast 2016;2016:9162590.

14. Koike E, Toda S, Yokoi F, Izuhara K, Koike N, Itoh K, Miyazaki K, Sugihara H. Expression of new human inorganic pyrophosphatase in thyroid diseases: its intimate association with hyperthyroidism. Biochem Biophys Res Commun 2006;341:691-6.

15. Altshuler LL, Bauer M, Frye MA, Gitlin MJ, Mintz J, Szuba MP, Leight KL, Whybrow PC. Does thyroid supplementation accelerate tricyclic antidepressant response? A review and meta-analysis of the literature. Am J Psychiatry 2001;158:1617-22.

16. Di Liegro I. Thyroid hormones and the central nervous system of mammals (Review). Mol Med Rep 2008;1:279-95.

17. Henrichs J, Bongers-Schokking JJ, Schenk JJ, Ghassabian A, Schmidt HG, Visser TJ, Hooijkaas H, de Muinck Keizer-Schrama SM, Hofman A, Jaddoe VV, Visser W, Steegers EA, Verhulst FC, de Rijke YB, Tiemeier H. Maternal thyroid function during early pregnancy and cognitive functioning in early childhood: the generation $\mathrm{R}$ study. J Clin Endocrinol Metab 2010;95:4227-34.

18. Duntas LH, Maillis A. Hypothyroidism and depression: salient aspects of pathogenesis and management. Minerva 
Endocrinol 2013;38:365-77.

19. Ittermann T, Volzke H, Baumeister SE, Appel K, Grabe HJ. Diagnosed thyroid disorders are associated with depression and anxiety. Soc Psychiatry Psychiatr Epidemiol 2015;50:1417-25.

20. Cooper-Kazaz R, Apter JT, Cohen R, Karagichev L, Muhammed-Moussa S, Grupper D, Drori T, Newman ME, Sackeim HA, Glaser B, Lerer B. Combined treatment with sertraline and liothyronine in major depression: a randomized, double-blind, placebo-controlled trial. Arch Gen Psychiatry 2007;64:679-88.

21. Almeida OP, Alfonso H, Flicker L, Hankey G, Chubb SA, Yeap BB. Thyroid hormones and depression: the Health in Men study. Am J Geriatr Psychiatry 2011;19:763-70.

22. Grabe HJ, Volzke H, Ludemann J, Wolff B, Schwahn C, John U, Meng W, Freyberger HJ. Mental and physical complaints in thyroid disorders in the general population. Acta Psychiatr Scand 2005;112:286-93.

23. Naicker M, Abbai N, Naidoo S. Bipolar limbic expression of auto-immune thyroid targets: thyroglobulin and thyroid-stimulating hormone receptor. Metab Brain Dis 2019;34:1281-98.

24. Li J, Donangelo I, Abe K, Scremin O, Ke S, Li F, Milanesi A, Liu YY, Brent GA. Thyroid hormone treatment activates protective pathways in both in vivo and in vitro models of neuronal injury. Mol Cell Endocrinol 2017;452:120-30.

25. Warner A, Mittag J. Thyroid hormone and the central control of homeostasis. J Mol Endocrinol 2012;49:R29-35.

26. Gilbert ME, Goodman JH, Gomez J, Johnstone AF, Ramos RL. Adult hippocampal neurogenesis is impaired by transient and moderate developmental thyroid hormone disruption. Neurotoxicology 2017;59:9-21.

27. Jessberger S, Gage FH. Adult neurogenesis: bridging the gap between mice and humans. Trends Cell Biol 2014;24:558-63.

28. Eriksson PS, Perfilieva E, Bjork-Eriksson T, Alborn AM, Nordborg C, Peterson DA, Gage FH. Neurogenesis in the adult human hippocampus. Nat Med 1998;4:1313-7.

29. Vern BA, Schuette WH, Leheta B, Juel VC, Radulovacki M. Low-frequency oscillations of cortical oxidative metabolism in waking and sleep. J Cereb Blood Flow Metab 1988;8:215-26.

30. Honey CJ, Sporns O, Cammoun L, Gigandet X, Thiran JP, Meuli R, Hagmann P. Predicting human resting-state functional connectivity from structural connectivity. Proc Natl Acad Sci U S A 2009;106:2035-40.

31. Wang Z, Dai Z, Gong G, Zhou C, He Y. Understanding structural-functional relationships in the human brain: a large-scale network perspective. Neuroscientist 2015;21:290-305.

32. Cao X, Liu Z, Xu C, Li J, Gao Q, Sun N, Xu Y, Ren Y, Yang C, Zhang K. Disrupted resting-state functional connectivity of the hippocampus in medication-naive patients with major depressive disorder. J Affect Disord 2012;141:194-203.

33. Tang Y, Kong L, Wu F, Womer F, Jiang W, Cao Y, Ren L, Wang J, Fan G, Blumberg HP, Xu K, Wang F. Decreased functional connectivity between the amygdala and the left ventral prefrontal cortex in treatment-naive patients with major depressive disorder: a resting-state functional magnetic resonance imaging study. Psychol Med 2013;43:1921-7.

34. Sheline YI. Depression and the hippocampus: cause or effect? Biol Psychiatry 2011;70:308-9.

35. Delaveau P, Jabourian M, Lemogne C, Guionnet S, Bergouignan L, Fossati P. Brain effects of antidepressants in major depression: a meta-analysis of emotional processing studies. J Affect Disord 2011;130:66-74.

36. Milne AM, MacQueen GM, Hall GB. Abnormal hippocampal activation in patients with extensive history of major depression: an fMRI study. J Psychiatry Neurosci 2012;37:28-36.

37. Fairhall SL, Sharma S, Magnusson J, Murphy B. Memory related dysregulation of hippocampal function in major depressive disorder. Biol Psychol 2010;85:499-503.

38. Kelley R, Garrett A, Cohen J, Gomez R, Lembke A, Keller J, Reiss AL, Schatzberg A. Altered brain function underlying verbal memory encoding and retrieval in psychotic major depression. Psychiatry Res 2013;211:119-26.

39. Ashburner J, Friston KJ. Voxel-based morphometry--the methods. Neuroimage 2000;11:805-21.

40. Chen L, Wang Y, Niu C, Zhong S, Hu H, Chen P, Zhang S, Chen G, Deng F, Lai S, Wang J, Huang L, Huang R. Common and distinct abnormal frontal-limbic system structural and functional patterns in patients with major depression and bipolar disorder. Neuroimage Clin 2018;20:42-50.

41. First MB, Gibbon M, Williams JBW. Structured Clinical Interview for DSM-IV Axis I \& II Disorders (Version 2.0). New York: New York State Psychiatric Institute, 1995.

42. Hamilton $M$. A rating scale for depression. J Neurol Neurosurg Psychiatry 1960;23:56-62.

43. Lish JD, Weissman MM, Adams PB, Hoven CW, Bird H. Family psychiatric screening instruments for epidemiologic 
studies: pilot testing and validation. Psychiatry Res 1995;57:169-80.

44. Ashburner J, Friston KJ. Unified segmentation. Neuroimage 2005;26:839-51.

45. Yan CG, Wang XD, Zuo XN, Zang YF. DPABI: Data Processing \& Analysis for (Resting-State) Brain Imaging. Neuroinformatics 2016;14:339-51.

46. Tzourio-Mazoyer N, Landeau B, Papathanassiou D, Crivello F, Etard O, Delcroix N, Mazoyer B, Joliot M. Automated anatomical labeling of activations in SPM using a macroscopic anatomical parcellation of the MNI MRI single-subject brain. Neuroimage 2002;15:273-89.

47. Polimanti R, Wang Q, Meda SA, Patel KT, Pearlson GD, Zhao H, Farrer LA, Kranzler HR, Gelernter J. The Interplay Between Risky Sexual Behaviors and Alcohol Dependence: Genome-Wide Association and Neuroimaging Support for LHPP as a Risk Gene. Neuropsychopharmacology 2017;42:598-605.

48. Tang Y, Wang F, Xie G, Liu J, Li L, Su L, Liu Y, Hu X, He Z, Blumberg HP. Reduced ventral anterior cingulate and amygdala volumes in medication-naive females with major depressive disorder: A voxel-based morphometric magnetic resonance imaging study. Psychiatry Res 2007;156:83-6.

49. Kong L, Chen K, Womer F, Jiang W, Luo X, Driesen N, Liu J, Blumberg H, Tang Y, Xu K, Wang F. Sex differences of gray matter morphology in cortico-limbic-striatal neural system in major depressive disorder. J Psychiatr Res 2013;47:733-9.

50. Zou K, Deng W, Li T, Zhang B, Jiang L, Huang C, Sun X, Sun X. Changes of brain morphometry in firstepisode, drug-naive, non-late-life adult patients with major depression: an optimized voxel-based morphometry study. Biol Psychiatry 2010;67:186-8.

51. Shad MU, Muddasani S, Rao U. Gray matter differences between healthy and depressed adolescents: a voxel-based morphometry study. J Child Adolesc Psychopharmacol 2012;22:190-7.

52. Hulvershorn LA, Cullen K, Anand A. Toward dysfunctional connectivity: a review of neuroimaging findings in pediatric major depressive disorder. Brain Imaging Behav 2011;5:307-28.

53. Kong L, Wu F, Tang Y, Ren L, Kong D, Liu Y, Xu K, Wang F. Frontal-subcortical volumetric deficits in single episode, medication-naive depressed patients and the effects of 8 weeks fluoxetine treatment: a VBM-DARTEL study. PLoS One 2014;9:e79055.

54. Schilbach L, Hoffstaedter F, Muller V, Cieslik EC,
Goya-Maldonado R, Trost S, Sorg C, Riedl V, Jardri R, Sommer I, Kogler L, Derntl B, Gruber O, Eickhoff SB. Transdiagnostic commonalities and differences in resting state functional connectivity of the default mode network in schizophrenia and major depression. Neuroimage Clin 2015;10:326-35.

55. Jin C, Gao C, Chen C, Ma S, Netra R, Wang Y, Zhang M, Li D. A preliminary study of the dysregulation of the resting networks in first-episode medication-naive adolescent depression. Neurosci Lett 2011;503:105-9.

56. Zhang J, Wang J, Wu Q, Kuang W, Huang X, He Y, Gong Q. Disrupted brain connectivity networks in drug-naive, first-episode major depressive disorder. Biol Psychiatry 2011;70:334-42.

57. Gong Q, He Y. Depression, neuroimaging and connectomics: a selective overview. Biol Psychiatry 2015;77:223-35.

58. Ye M, Yang T, Qing P, Lei X, Qiu J, Liu G. Changes of Functional Brain Networks in Major Depressive Disorder: A Graph Theoretical Analysis of Resting-State fMRI. PLoS One 2015;10:e0133775.

59. Peng D, Shi F, Shen T, Peng Z, Zhang C, Liu X, Qiu M, Liu J, Jiang K, Fang Y, Shen D. Altered brain network modules induce helplessness in major depressive disorder. J Affect Disord 2014;168:21-9.

60. Bora E, Fornito A, Pantelis C, Yucel M. Gray matter abnormalities in Major Depressive Disorder: a metaanalysis of voxel based morphometry studies. J Affect Disord 2012;138:9-18.

61. Geng H, Wu F, Kong L, Tang Y, Zhou Q, Chang M, Zhou Y, Jiang X, Li S, Wang F. Disrupted Structural and Functional Connectivity in Prefrontal-Hippocampus Circuitry in First-Episode Medication-Naive Adolescent Depression. PLoS One 2016;11:e0148345.

62. Alvarez JA, Emory E. Executive function and the frontal lobes: a meta-analytic review. Neuropsychol Rev 2006;16:17-42.

63. Zhang L, Zhang L, Xue F, Yue K, Peng H, Wu Y, Sha O, Yang L, Ding Y. Brain morphological alteration and cognitive dysfunction in multiple system atrophy. Quant Imaging Med Surg 2018;8:1030-8.

64. Le Bihan D, Mangin JF, Poupon C, Clark CA, Pappata S, Molko N, Chabriat H. Diffusion tensor imaging: concepts and applications. J Magn Reson Imaging 2001;13:534-46.

65. Wang L, LaBar KS, Smoski M, Rosenthal MZ, Dolcos F, Lynch TR, Krishnan RR, McCarthy G. Prefrontal mechanisms for executive control over emotional distraction are altered in major depression. Psychiatry Res 
2008;163:143-55.

66. Raichle ME, MacLeod AM, Snyder AZ, Powers WJ, Gusnard DA, Shulman GL. A default mode of brain function. Proc Natl Acad Sci U S A 2001;98:676-82.

67. Chen YC, Zhang H, Kong Y, Lv H, Cai Y, Chen H, Feng Y, Yin X. Alterations of the default mode network and cognitive impairment in patients with unilateral chronic tinnitus. Quant Imaging Med Surg 2018;8:1020-9.

68. Strobl MJ, Freeman D, Patel J, Poulsen R, Wendler CC, Rivkees SA, Coleman JE. Opposing Effects of Maternal Hypo- and Hyperthyroidism on the Stability of Thalamocortical Synapses in the Visual Cortex of Adult Offspring. Cereb Cortex 2017;27:3015-27.

69. Zhu X, Li R, Wang P, Li J. Aberrant functional connectivity of the hippocampus in older adults with subthreshold depression. Psych J 2014;3:245-53.

Cite this article as: Cui L, Wang F, Yin Z, Chang M, Song Y, Wei Y, Lv J, Zhang Y, Tang Y, Gong X, Xu K. Effects of the LHPP gene polymorphism on the functional and structural changes of gray matter in major depressive disorder. Quant Imaging Med Surg 2020;10(1):257-268. doi: 10.21037/ qims.2019.12.01
70. Aston C, Jiang L, Sokolov BP. Transcriptional profiling reveals evidence for signaling and oligodendroglial abnormalities in the temporal cortex from patients with major depressive disorder. Mol Psychiatry 2005;10:309-22.

71. Chen J, Xu Y, Zhang J, Liu Z, Xu C, Zhang K, Shen Y, Xu Q. Genotypic association of the DAOA gene with restingstate brain activity in major depression. Mol Neurobiol 2012;46:361-73.

72. McEwen BS. Stress, sex, and neural adaptation to a changing environment: mechanisms of neuronal remodeling. Ann N Y Acad Sci 2010;1204 Suppl:E38-59.

73. Delgado y Palacios R, Verhoye M, Henningsen K, Wiborg O, Van der Linden A. Diffusion kurtosis imaging and high-resolution MRI demonstrate structural aberrations of caudate putamen and amygdala after chronic mild stress. PLoS One 2014;9:e95077. 


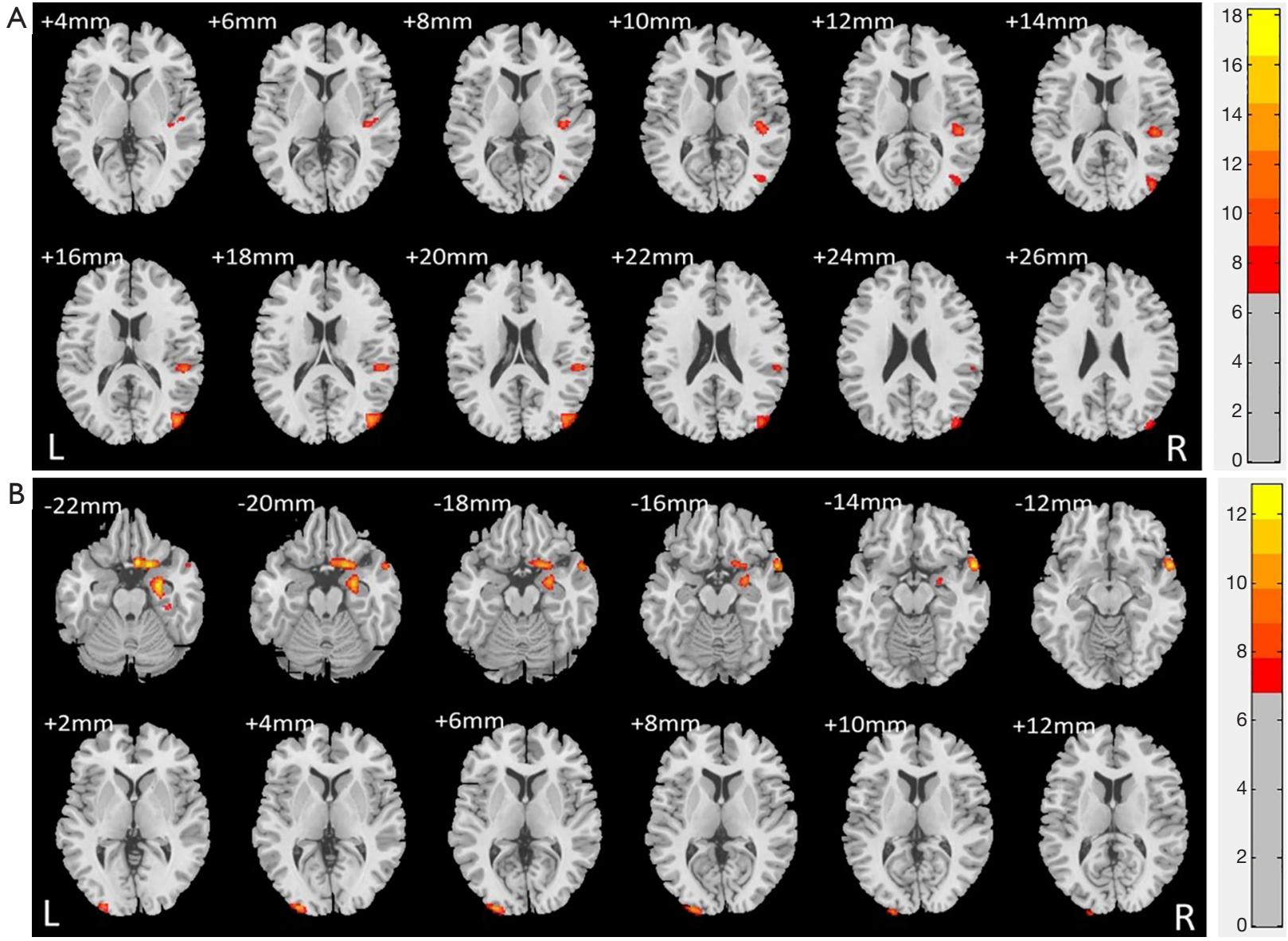

Figure S1 Brain regions with: (A) significant diagnosis main effects on GMV; (B) significant genotype main effects on GMV. GMV, gray matter volume. 